\title{
Horizontal diffusion by submeso motions in the stable boundary layer
}

\author{
L. Mahrt • R. Mills
}

Received: 30 October 2008 / Accepted: 25 February 2009 / Published online: 13 March 2009

(C) The Author(s) 2009. This article is published with open access at Springerlink.com

\begin{abstract}
Four networks of wind data are used to construct the first systematic estimates of the horizontal diffusivity from observations of submeso motions on scales often unresolved in numerical models. Currently, the horizontal diffusivity in numerical models is specified mainly for numerical reasons without observational support. The data analysis in this study emphasizes the stable boundary layer although results are briefly presented for the unstable boundary layer. The horizontal diffusivity is estimated from the horizontal gradient and the observed flux. Horizontal gradients of scalars are generally difficult to directly estimate from observations with sufficient accuracy for much of the data. As an alternative, simulated particles with conservative properties are introduced into the observed wind field in order to estimate the horizontal diffusivity for submeso motions. The sensitivity of the horizontal diffusivity to details of the method is examined. The horizontal diffusivity increases with the range of time and space scales that are included in the evaluation. The horizontal diffusivity is much larger with significant topography and may increase with wind speed, depending on the site location. The coarse station spacing or the small domain size is found to be a major limitation to the analysis.
\end{abstract}

Keywords Dispersion $\cdot$ Diffusion $\cdot$ Stable boundary layer $\cdot$ Submeso $\cdot$ Mesoscale

\section{Introduction}

The horizontal transport by atmospheric mesoscale motions has been studied mainly for special cases of well-defined phenomena such as thunderstorms, sea breezes, and slope winds. In boundary layers with strong stratification where turbulence mixing is weak, horizontal transport by mesoscale motions becomes important [22]. Based on numerical studies,

\footnotetext{
L. Mahrt $(\bowtie) \cdot$ R. Mills

NorthWest Research Associates, Redmond, WA 98009, USA

e-mail: mahrt@nwra.com; mahrt@coas.oregonstate.edu

L. Mahrt

Oregon State University, Corvallis, USA
} 
laboratory experiments and theoretical considerations, the mesoscale modes in stratified flow are viewed as a combination of a variety of types of gravity waves $[13,14,17,19]$; horizontal modes sometimes referred to as pancake vortices [16]; solitons [20]; and a wide variety of other complex forms (see references in [9]). In the daytime convective boundary layer, the small-scale part of the mesoscale range is replaced by turbulent boundary-layer scale eddies, which might include convectively driven boundary-layer eddies and longitudinal roll vortices e.g., $[4,8]$. Such large eddies may be coupled to gravity waves above the boundary layer sometimes referred to as convection waves e.g., [6]. Since daytime "turbulent" eddies extend to much larger scales compared to turbulence in the stable boundary layer, the important daytime mesoscale motions begin at larger scales compared to the mesoscale motions in the stable boundary layer.

The nocturnal mesoscale motions included in this study extend down to Eulerian time scales of a minute or less, horizontal length scales as small as ten metres and vertical depth scales as small as several metres. To reduce conflicts with traditional use of the term "mesoscale", we use the term "submeso" to describe motions extending down to such small scales e.g., $[2,3,11]$. In particular, we pragmatically refer to all of the motions resolved by the networks as "submeso", without specific physical meaning. In this regard, we make an analogy between the networks and grid boxes of a numerical model. Then "submeso" in a numerical model becomes analogous to "unresolved".

In numerical models, the unresolved horizontal transport between grid cells is represented by a single horizontal diffusivity for turbulence and unresolved submeso motions combined. Presently, there is no observational basis for determination of the horizontal diffusivity. The numerical values of such diffusivities have historically been determined by numerical considerations in order to control numerically unstable modes, particularly "two-delta-x waves". The total model horizontal diffusion is the sum of the implicit numerical diffusion in the differencing scheme and the explicit horizontal diffusion related to the horizontal diffusivity.

The horizontal flux has been generally neglected in boundary layer studies because the horizontal divergence of the flux occurs on much larger horizontal scales than the vertical scale of the vertical flux divergence. However, with strongly stratified flow, the vertical mixing of scalars by the turbulence may become extremely weak e.g., [25] while the horizontal transport by submeso motions can be rather large, as found in this study. The vertical flux by the submeso motions is difficult to estimate due to large sampling errors and difficulties measuring very weak vertical motions, and is outside the scope of this study.

Theoretically, relating the horizontal flux to the horizontal gradient is uncertain. Important horizontal flux can occur through rotation of the vertical flux in the presence of vertical shear that is not related to the mean horizontal gradient [5,24]. For example, horizontal velocity fluctuations are significantly correlated with fluctuations of temperature and other quantities because vertical velocity fluctuations systematically generate fluctuations of both horizontal velocity and scalars. In stratified flow, downdrafts (updrafts) generate positive (negative) temperature fluctuations and positive (negative) along-wind velocity fluctuations. As a result, horizontal flux of heat can be generated without horizontal gradients of temperature. Fortunately, this process may be less important for submeso motions in stratified flow, compared to turbulence, because the submeso vertical motions are generally either very weak or associated with wave motions with little vertical scalar flux.

No attempt known to us has been made to directly estimate horizontal diffusivities for transport by submeso motions using networks of observations. Unfortunately, horizontal gradients of scalars in the stable boundary layer are normally too erratic for reliable estimates except in special cases [12]. Therefore, we will examine the horizontal diffusivity estimated from simulated particles released into the observed wind field. This study emphasizes the 
stable case where the horizontal diffusion by submeso motions is thought to play a greater relative role compared to the daytime convective boundary layer.

\section{Data analysis}

Approximately 30 networks were examined for possible use for estimation of the horizontal diffusivity. All but three were eliminated because of inadequate sampling frequency, inadequate instrument response time, erratic station spacing, nonuniform deployment or inadequate documentation. The three selected networks still include major shortcomings for the analyses needed here. We confine the nocturnal computations to the 10-h period from 2000 to 0600 local time.

The CASES99 network [15] consists of 7 sonic anemometers deployed $5 \mathrm{~m}$ above the ground surface across a 600-m wide domain with short grass ground cover. The Iowa network [7] deployed 12 sonic anemometers above corn and soybeans. Here, we exclude the three eastern sonic anemometers, which are missing significant data. This elimination also reduces the ratio of the east-west network width to the north-south width from almost 3 to about 1.5. A ratio of unity is optimal.

The Utah network (Dugway Proving Ground) consists of 26 stations with R. M. Young Model 05103 anemometers with an accuracy of $0.3 \mathrm{~ms}^{-1}$ and an official startup threshold of $1.0 \mathrm{~ms}^{-1}$. Numerous values of $<1.0 \mathrm{~ms}^{-1}$ were observed possibly due to a smaller threshold for cessation of propeller motion. In addition, the propeller could be motionless for only part of the 5 -min summation periods. About $0.2 \%$ of the 5 -min periods reported zero motion. We do not eliminate these infrequent cases. This network is characterized by large station spacing of 5-10 km such that much of the submeso motion on time scales $<5 \mathrm{~min}$ are not spatially resolved. To partially compensate for the coarser time resolution, the wind components are linearly interpolated to 1-min intervals. The linear interpolation leads to only minor changes in the computed values of the horizontal diffusivity.

The two-dimensional horizontal nature of the motions in stratified flow is problematic with terrain features between stations. For example, a low range of hills might preclude horizontal transport across the range and the correlation of the wind field between adjacent stations might be small. Or, air motion at a station may be confined to pools of cold air trapped in low-lying areas, not resolved by the network. In these cases, the network winds may overestimate the horizontal dispersion since the interpolated wind field does not include interruption of surface airflow by trapping cold air in low lying areas. The Utah network includes several ranges of hills and mountains. Since the wind interpolation scheme used below ignores such interruption of the surface flow, we define two subnetworks that are not disrupted by significant topography. This seriously reduces the number of stations. For the Utah network, this paper will focus on a subnetwork in the western half of the main basin (Fig. 1). A second "northwest" Utah network is defined, but will be used less since the between-station spacing is generally greater than $10 \mathrm{~km}$. More information on the networks can be found in Mahrt et al. [10].

\subsection{Wind field}

To filter out the turbulence for stable conditions, the flow is first averaged over 1-min periods at each station. Based on cospectra, the turbulence is normally confined to scales $<1 \mathrm{~min}$ for our stable boundary-layer data. The 1-min averages can sometimes exclude the smallest submeso motions, but could inadvertently include some turbulence in the windiest cases. 


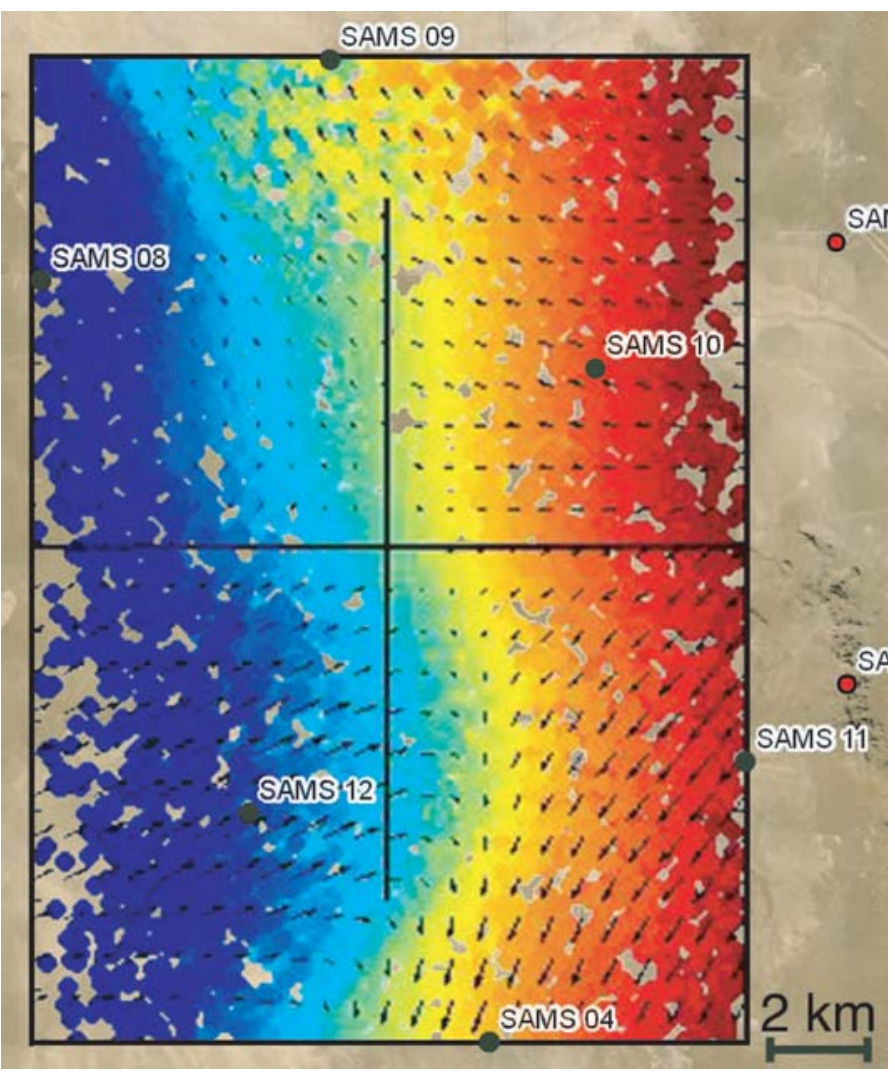

Fig. 1 The Utah subnetwork is defined by the square box. This subnetwork includes only 6 of the original 26 stations in an effort to avoid to horizontal decoupling by terrain features. The horizontal and vertical lines intersecting the centre are the sampling lines. The simulation in this figure uses the north-south sampling line where the initial isolines of $q$ are in the north-south direction (Sect. 4). The simulated particles are colour coded according to their value of $q$ in arbitrary units. This figure is an example of particle displacement after $1 \mathrm{~h}$ of simulation. Patterns vary dramatically between 1 -h periods

Alternatively, one could partition the turbulence and submeso motions for each individual record; however, we forego application of a variable averaging time in favour of examination of a constant range of scales.

Stochastically, the time scale of the submeso motion increases approximately linearly with its horizontal scale, but at a rate that is an order of magnitude smaller than Taylors hypothesis [10], as sketched in Fig. 2. This implies that propagation velocities are much larger than the wind speed. In addition, the propagation direction based on animations of the wind field often showed little relation to the wind direction. For individual motions, deviations from the mean time-space relationship in Fig. 2 are large. Since each network resolves different spatial scales, optimum time averaging of flow statistics might be chosen in terms of the network size and station spacing. However, this procedure is complex and the relationship between space and time scales is not sufficiently robust for confident choices of time scales. Therefore, we average over a constant time period, here chosen to be $1 \mathrm{~h}$. Thus, we include motions between time scales of $1 \mathrm{~min}$ and $1 \mathrm{~h}$ and on horizontal scales between the station spacing and the domain size, as sketched in Fig. 2. 


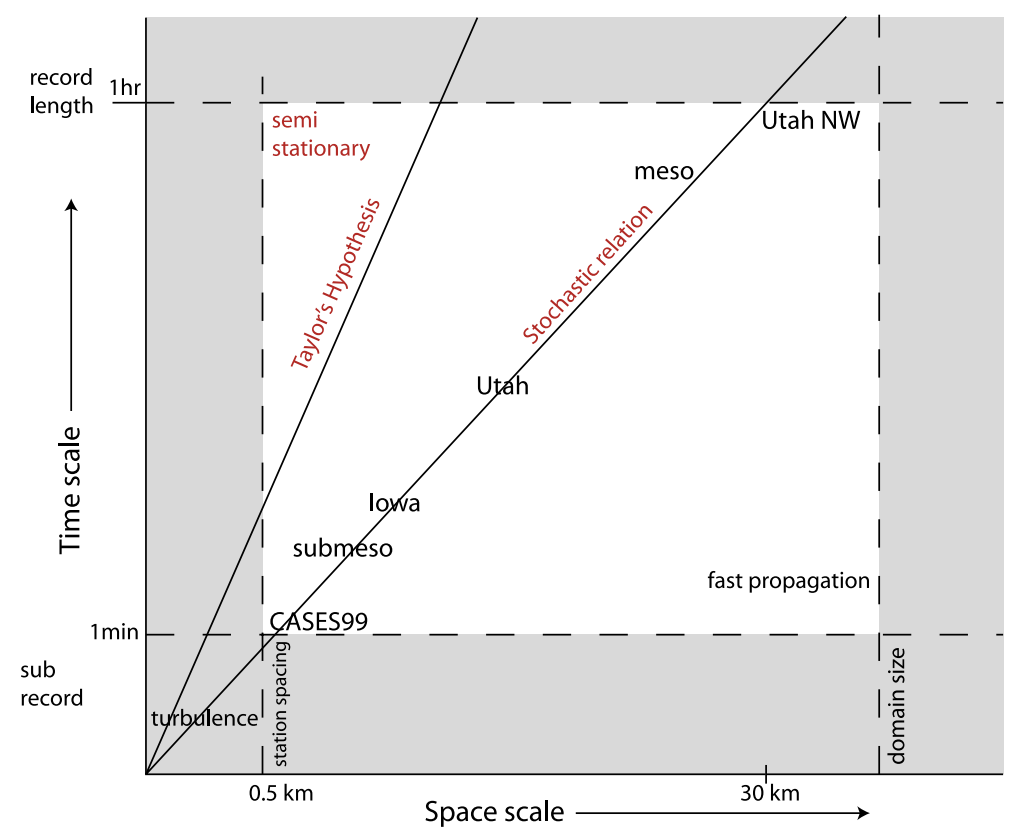

Fig. 2 Schematic of space and time scales defining the present analysis. The stochastic line (space scale $=8.3 \mathrm{~ms}^{-1}$ time scale) represents the average relation between space and time scales for mesoscale motions in Mahrt et al. [10]. The nocturnal wind speed averaged over all of the nocturnal datasets is approximately $2.5 \mathrm{~ms}^{-1}$. For individual records, the deviations from this line are large. The outer grey area indicates the exclusion of time scales through choice of averaging and record length, and exclusion of horizontal scales due to the finite station spacing and size of the network

For application to modelling, we make an analogy between the entire network and a model grid area by considering the network-averaged wind to be the model resolved flow. We will remove the network-averaged wind from the 1-min observations to focus on smaller-scale (subgrid) motions. Removal of the network-averaged wind can be viewed as transforming the coordinate system to move with the spatially averaged flow. Larger mesoscale motions dominate the time dependence of the network-averaged wind so that the removal of the network-averaged flow partially removes time variations on larger time scales. This was quantitatively examined by Belušić and Mahrt [3] who studied the influence of removing the spatial mean on sequentially smaller scales. In the present study, the removal of the network mean does not influence the horizontal gradients of wind within the network.

The network-averaged wind is computed by first interpolating the station winds to a finer grid using inverse square weighting and then spatially averaging the gridded wind vectors. This reduces the direct bias introduced by simple averaging of the stations winds with nonuniform distribution of station locations. The grid spacing was $50 \mathrm{~m}$ for the CASES99 network, $500 \mathrm{~m}$ for the Iowa network and $1 \mathrm{~km}$ for the Utah network. To further reduce the bias in the Iowa network, a cluster of 4 stations were averaged to form one effective station.

\subsection{Unstable case}

Estimation of the horizontal diffusivity for the daytime unstable case incurs additional difficulties. The large daytime boundary-layer eddies appear to be as large as the entire CASES99 
network; therefore, CASES99 is excluded from the daytime analysis. Ten-minute averaging of the data will be used instead of 1-min averaging in order to filter out the turbulence, which occurs on larger time scales compared to the nocturnal turbulence. This increased averaging of the initial data reduces the sample size by a factor of 10 compared to 1-min averages. For this reason, we extend the flux averaging period from 1 to $4 \mathrm{~h}$. Extending the averaging time to even longer time periods incorporates significant nonstationarity due to diurnal variation. In fact, we limit our analysis to a single 4-h period each day from 1100 to 1500 local time in order to minimize diurnal variation. The resulting sample sizes are marginal.

\section{Horizontal diffusivity}

Computing the horizontal diffusivity from observed horizontal fluxes is problematic because estimation of the horizontal gradients from the data is generally prone to large errors. The horizontal gradients of scalars, such as temperature and moisture, are only occasionally well-defined in our networks and generally sensitive to the method and scale of the gradient estimate. Unfortunately, estimates of the behaviour of the horizontal transport require a large sample size and do not permit limiting the analysis to special selected cases with welldefined horizontal gradients. Application of smoothing techniques to improve the estimate of the horizontal gradients was too sensitive to the choice of method.

Alternatively, the horizontal diffusivity can be estimated by releasing simulated particles into the observed wind field. Simulated particles are released into the flow with the conserved value of a generic scalar, $q$, determined from the particle release point. The goal here is to use the released particles as an intermediate tool to quantify the horizontal transport by the observed submeso motions rather than to propose a particle model for general use, as in [23]. The current approach is somewhat analogous to semi-Lagrangian advection schemes in numerical models e.g., [18] except here we are using observed winds that are interpolated to each particle position that then transports the particle over a given time step.

The source value of the generic scalar is specified to increase linearly in the $x$-direction with no dependence on the $y$-direction, such that

$$
\overline{q_{s f c}}(x)=\frac{\mathrm{d} \overline{q_{s f c}}}{\mathrm{~d} x} x
$$

where $x$ is the distance of the particle release point from the west edge of the domain and the gradient is assumed to be constant in space and time.

The simulations initially release 2,000 particles randomly distributed throughout the network domain. Particle velocities are updated each minute. To offset loss of particles across the domain boundary during the iteration, an additional 100 particles are released each minute with random spatial distribution. Integrations are carried out over each of the 1-h periods that comprise the 10-h nocturnal period. The particles transported by the observed flow lead to a horizontal flux of $q$. The motion of the particles is computed for each one-minute period using inverse-square interpolation of the gridded winds to each particle position.

While this approach leads to more stable estimates of the horizontal diffusivity compared to estimating horizontal gradients from the observations, several important limitations remain:

(1) The simulations are two-dimensional with no vertical transport.

(2) Removal of the domain-averaged flow to simulate removing the model resolved flow (Sect. 2.1) implies that the particle simulation grid is moving with the mean flow, yet 
the input wind data are from stations at fixed points. Therefore, the present approach is only a tool to isolate the transport by submeso motions and reduce the depletion of simulated particles from rapid transport out of the domain by the mean flow.

(3) The spatial structure of the smallest submeso variation is lost due to the limited spatial resolution of the networks. This is a serious deficiency.

(4) Horizontal gradients of the density of the particles may develop and influence the value of the flux across the sampling line. Sensitivity tests suggest that this difficulty is somewhat random and much less important than the problem of network resolution.

(5) A large sample size is required to stochastically describe the impact of submeso motions, partly because of their larger time scale compared to the turbulence eddies and the significant influence of isolated submeso events. The sample sizes for the Iowa and CASES99 networks are a significant limitation to the analysis.

(6) The larger networks spatially resolve some motions with time scales greater than $1 \mathrm{~h}$, which could lead to overestimation of the transport.

As an example of the last difficulty, the initial phase of a low-frequency wave mode leads to gradient transport since any motion perpendicular to the sampling line initially generates gradient flux. Even if the total horizontal flux over an entire period of a wave is zero, the last part of the cycle may not be completely included in the 1-h period and, therefore, the total horizontal flux may be artificially increased. Such a wave mode seems to be developing in Fig. 1, however the "return phase" of this wave was not subsequently observed, resulting in a true net flux even with longer averaging. This sampling problem is briefly examined in the next section. Because of the above six difficulties, the errors for the horizontal diffusivities will be large and the error estimates themselves are uncertain (Sect. 5).

\section{Decomposition}

To compute the transport of $q$ by the particle motion, we define a sampling line through the centre of the domain to simulate the wall of a model grid box. The particle velocity and value of $q$ is recorded as each particle crosses the sampling line. Sampling lines are defined in the north-south direction with specified east-west scalar gradients (Fig. 1) and in the east-west direction for specified north-south gradients. The length of the sampling line is equal to the length of the smaller of the east-west and north-south widths of the observational domain. For brevity, we now discuss the decomposition only in terms of the north-south sampling line. Recall that the network averaged wind has been removed.

To obtain a larger sample, the particles crossing the sampling line are collected over a time period, $\tau_{F}$, here $1 \mathrm{~h}$. We then decompose the particle flow as

$$
\phi=\bar{\phi}+\phi^{*}
$$

where $\phi$ is the particle value of either $q$ or $u$ when the particle crosses the sampling line, the overbar is the 1-h average for all of the particle values crossing the sampling line within a 1-h record, and symbol * indicates the deviations of the individual particle values from this average. We view this averaging as an approximation to an ensemble average even if a poor one with large sampling errors. The overbar averaging operator implicitly includes spatial averaging along the sampling line in addition to 1-h time averaging. We assume that the statistics are independent of position along the north-south sampling line since the generation of particle $q$-values is independent of $y$. This assumption requires that variations of the wind 
along the north-south line are random. The error due to heterogeneity of the wind field is discussed below.

The submeso flux of $q$ across the sampling line over time interval $\tau_{F}$ is

$$
\overline{u^{*} q^{*}} \text {. }
$$

Since $q^{*}$ is generated by velocity fluctuations, the flux tends to increase faster than linearly with increasing strength of the submeso motions $\left(\sigma_{u^{*}}\right)$ where $\sigma_{u^{*}}$ refers to the standard deviation of the particle velocities at the sampling line during the 1-hr period. However, the correlation between $u^{*}$ and $q^{*}$ is small, averaging a little more than 0.2 for the Iowa network, a little more than 0.3 for the CASES99 network and about 0.6 for the Utah network. It is not known if the more significant correlation for the Utah network is due to inclusion of larger spatial scales or organization of motions by the topography.

Since sampling errors are large for individual 1-h records, we subsequently average the fluxes over a longer period, here, over all of the nocturnal records for the entire field program. This flux is written as

$$
\left\langle\overline{u^{*} q^{*}}\right\rangle
$$

where the angle bracket denotes averages for the entire field program, recorded in Table 1.

Table 1 Networks

\begin{tabular}{cllllll}
\hline Site & Net. size & $K_{H \text { sub }}$ & $\mathrm{SE}_{\text {sub }}$ & $-R_{u q}$ & $\sigma_{u}$ & $\sigma_{q}$ \\
\hline Iowa & 5.7 & $\mathbf{8 . 5}$ & 0.12 & 0.21 & 0.19 & 2.00 \\
Short & 5.7 & 8.1 & 0.12 & 0.22 & 0.19 & 1.99 \\
10h & 5.7 & 4.9 & 0.10 & 0.21 & 0.18 & 1.30 \\
FP & 5.7 & 7.9 & 0.12 & 0.22 & 0.19 & 1.91 \\
EW & 5.7 & 22.7 & 0.08 & 0.33 & 0.19 & 3.29 \\
CASES99 & 0.5 & $\mathbf{4 . 5}$ & 0.09 & 0.37 & 0.16 & 0.64 \\
10h & 0.5 & 4.5 & 0.09 & 0.37 & 0.21 & 0.64 \\
FP & 0.5 & 6.4 & 0.07 & 0.37 & 0.21 & 0.73 \\
EW & 0.5 & 10.7 & 0.10 & 0.46 & 0.22 & 0.87 \\
Utah & 11 & $\mathbf{4 9 3}$ & 0.07 & 0.59 & 0.73 & 8.56 \\
Short & 11 & 201 & 0.10 & 0.46 & 0.44 & 6.40 \\
$10 \mathrm{~h}$ & 11 & 489 & 0.07 & 0.59 & 0.73 & 8.57 \\
FP & 11 & 460 & 0.08 & 0.59 & 0.73 & 7.95 \\
1 year & 11 & 654 & 0.02 & 0.62 & 0.81 & 9.54 \\
EW & 11 & 545 & 0.10 & 0.60 & 0.67 & 9.62 \\
UtahNW & 43 & $\mathbf{8 0 9}$ & 0.08 & 0.57 & 0.83 & 12.43 \\
\hline
\end{tabular}

The network size $(\mathrm{km})$, horizontal diffusivity $\left(\mathrm{m}^{2} \mathrm{~s}^{-1}\right)$, relative error, correlations between $u^{*}$ and $q^{*}$ and the standard deviations of $u\left(\mathrm{~ms}^{-1}\right)$ and $q$ (arbitrary units). "Short" refers to the reduction of the sampling line to $1 / 3$ of its original size. The network size is the smallest of the widths in the N-S and E-W directions. 10 year indicates removal of the wind vectors averaged over the 10-h nocturnal period, FP signifies removal of the wind vectors averaged over the entire field program, EW indicates use of the east-west oriented sampling lines and 1 year indicates using 1 year of data instead of 1 month of data for the Utah network. Bold is the eddy diffusivity value for the default computation 
The corresponding horizontal diffusivity for the submeso flow for the entire field program is computed as

$$
K_{H \text { sub }}=-\frac{\left\langle\overline{u^{*} q^{*}}\right\rangle}{\mathrm{d} \bar{q} / \mathrm{d} x} .
$$

Since the specified horizontal gradient is independent of time, the brackets are not needed in the denominator. The values in Table 1 suggest that the horizontal diffusivity increases with domain size and increases substantially with topography, explored in more detail in Sect. 6. Since our general approach has not been previously used, we first examine the dependence of the diffusivity on the calculation method.

\section{Sensitivity to method details}

To examine the sensitivity of the value of the horizontal diffusivity to the details of the method of calculation, we now examine a sequence of temporary alterations to the method.

\subsection{Sampling line}

The position of the sampling line within the network leads to differences in the horizontal diffusivity up to a factor of two, presumably due partly to the sparse station spacing in the presence of surface heterogeneity. Here, we have reported only the results for the sampling lines bisecting the centre of the network, which is least affected by network boundaries.

Decreasing the length of the sampling line captures a smaller range of spatial scales and is expected to decrease the horizontal diffusivity. Decreasing the sampling line to $1 / 3$ its original length reduces the diffusivity by more than half of its original value for the Utah network, but reduces the diffusivity by only $5 \%$ for the Iowa network. The CASES99 network is too small to further decrease the length of the sampling line.

The above results are based on a north-south sampling line. With an east-west sampling line in the Iowa network, the value of the diffusivity is more than twice that for the northsouth sampling line (Table 1). This augmentation may be due to the rectangular shape of the network with an east-west width about $50 \%$ greater than the north-south width. This would suggest that the network configuration is more important than the details of the computation method. However, the data also include large east-west variations of the $v$ component on some of the nights, which would increase the diffusivity for the east-west sampling line. The cause of such motions is not known. The horizontal diffusivity for the CASES99 network based on the east-west sampling line is about twice as large as that for the north-south sampling line. It is not known if this difference is related to the dominance of southerly flow in the CASES99 field program and the potential influence of the mean flow on the structure of the submeso flow. For the Utah network, the diffusivity with the east-west sampling line is about $5 \%$ greater than that for the north-south sampling line. Although this difference is of marginal significance, we also note that the Utah network experiences more north-south flow than east west flow, and is embedded in primarily north-south oriented topography.

\subsection{Removal of the stationary wind}

The wind field is influenced not only by transient submeso motions, but also by surface heterogeneity, instrument bias and micrometeorological siting of the instruments. To estimate the collective effect of these influences on the computed diffusivity, we have averaged the wind 
vectors at each station over a longer period and then removed this mean vector from the wind data at each station. This mean spatial variability is disorganized in the Iowa network. Perhaps each station is influenced by local surface conditions. However, the differences of the wind vector between stations could not be related to local crop type or row orientation. The experiment-averaged spatial variability of the wind field for the CASES99 network is related to very small local changes in surface elevation. The spatial variation across the network is most organized for the Utah network. The removal of the wind velocities averaged over the entire field program (Table 1, FP) reduces the horizontal diffusivity by only about 6-7\% for the Iowa and Utah networks. The removal of the experiment means actually increases the diffusivity for the CASES 99 network by about $40 \%$ suggesting that the surface heterogeneity partially counteracts horizontal diffusion associated with transient submeso motions, for unknown reasons.

The removal of the 10-h averaged winds filters out the night-to-night variability of the mean circulation and removes the influence of the micro-siting that varies from night to night due to wind direction changes. This removal reduces the submeso diffusivity by almost $40 \%$ for the Iowa network and a little more than $15 \%$ for the Utah network, but had no impact on the diffusivity for the CASES99 network. Evidently, the impact of removal of the stationary part of the flow on the horizontal diffusivity depends on the network; general conclusions are not possible.

\subsection{Averaging time}

The above calculations include motions between $1 \mathrm{~min}$ and $1 \mathrm{~h}$. To isolate the influence of the larger time scales, we have removed the 10-min averaged flow computed from nonoverlapping windows. This removal of motions on time scales $<10$ min reduces the horizontal diffusivity by about $20 \%$, the exact decrease varying with network and calculation method. This time averaging reduces the flux error (6) in Sect. 5.5. Such averaging may be preferable in terms of sampling errors, but introduces sensitivity of the diffusivity to the exact scales removed.

\subsection{The between-network variation}

The sensitivity of the horizontal diffusivity to the details of the method of calculation indicates that the diffusivity is computed with at least a factor of two uncertainty. Like the diffusivity itself, this sensitivity varies substantially between networks. Notice that the sensitivity of the diffusivity to the different methods listed in Table 1 for the Iowa and CASES99 networks occurs mainly through changes of $\sigma_{q}$ while the magnitude of the variability of the wind field $\sigma_{u}$ is less sensitive to method changes. This result implies that the different methods lead to different particle displacement statistics mainly because the value of $q$ at the sampling line is determined by the perpendicular particle travel distances from the particle origin. This in turn implies that the different calculation methods emphasize different horizontal scales. For the Utah network, the horizontal diffusivity is more sensitive to the variation of $\sigma_{u}$, compared to the other two networks, thus preventing general conclusions.

\subsection{Random error}

The randomness in the initial particle positions leads to small order of $1 \%$ differences between simulations. A more important random error occurs due to large differences between hourly records and the finite number of hourly records. The significance of the diffusivities can be assessed by estimating the relative standard error of the flux 


$$
\mathrm{SE}=\frac{\sigma_{F}}{|F| \sqrt{(N)}}
$$

where $F$ is the one-hour flux, $\sigma_{F}$ is the between-hour variation of $F,|F|$ is the absolute value of the flux averaged over the entire field program and $N$ is the number of one-hour records. The relative standard error does not qualify in terms of a random ensemble process since the one-hour records come from a variety of synoptic regimes. However, SE will serve as a relative measure of variability and uncertainty of the mean flux. This error measure is typically $10 \%$ for the submeso flux, suggesting that the flux values averaged over the entire field program are statistically significant. The relative standard error is small compared to the other uncertainties discussed above. Using the entire year of data for the Utah network decreases the relative standard error to only $2 \%$ and also increases the averaged diffusivity by about $30 \%$, partly due to stronger winds during the winter compared to the month of June selected for this study.

\section{Dependence on scale and ambient conditions}

The diffusivity presumably depends on both time and spatial variations of the flow. Anfossi et al. [1] and Vickers and Mahrt [21] found that the kinetic energy of the mesoscale motions based on time variation did not depend significantly on wind speed or stability. However, information on time variation cannot be used to infer spatial variation. For the Iowa network, the horizontal diffusivity for winds $>2 \mathrm{~ms}^{-1}$ is about twice that for winds $<2 \mathrm{~ms}^{-1}$, although the scatter is large (Fig. 3). The ratio of the averaged strong-wind horizontal diffusivity to the weak-wind horizontal diffusivity is about 1.6 for the Utah network, 1.2 for the CASES99 network, and near unity for the sparse Utah Northwest network. The large scatter and negative values of the diffusivity for some of the records (Fig. 3) result partly from the fact that the sampling problem for submeso motions is more severe than that for the turbulence, which occurs on smaller spatial scales (greater sample size). In addition, some or most of the types of submeso motions may not be augmented by wind speed. The cause of potential augmentation is not known although stronger wind speed corresponds to stronger

Fig. 3 Dependence of the hourly submeso diffusivity on the speed of the network-averaged wind vector for the Iowa Network. The large scatter is partly due to inadequate sampling of the submeso scale motions for individual hours. The large scatter may mask an increase of the horizontal diffusivity with increasing speed. The scatter is even greater for the other three networks

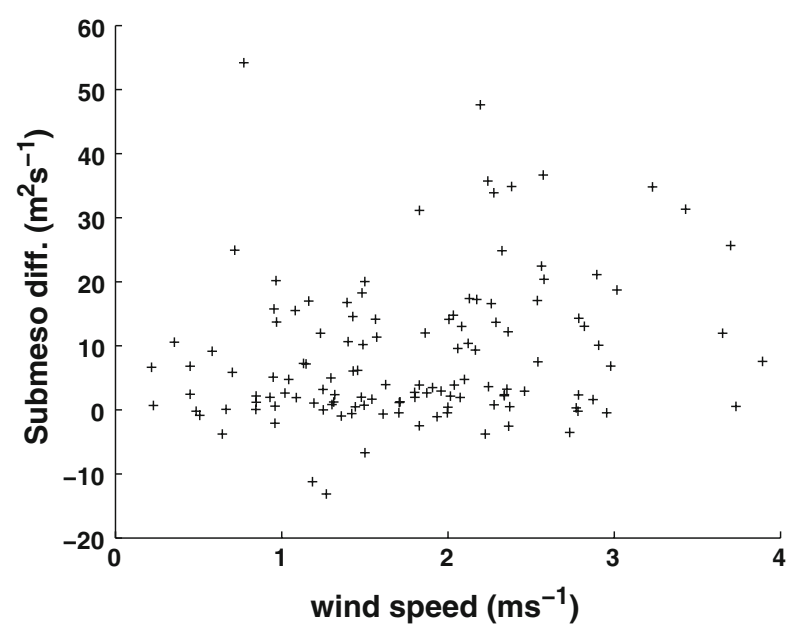




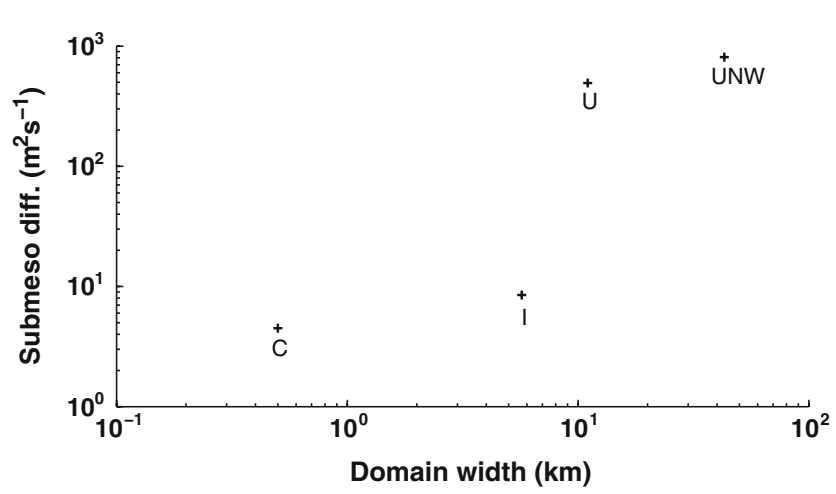

Fig. 4 The increase of the horizontal diffusivity with the size of the network based on averaged values of the horizontal flux for the CASES99 (C), Iowa (I), Utah (U) and Utah Northwest (UNW) networks

shear instability and possibly more gravity wave activity. For the strongest wind cases, the turbulence might directly contribute to some of the wind variance on time scales $>1 \mathrm{~min}$.

The horizontal diffusivity probably increases with the range of scales captured by the network (Fig. 4), consistent with the increase of the horizontal diffusivity with the network size for the 4 networks in Table 1. However, the diffusivity increase by about two orders of magnitude between the two smaller networks and the two Utah networks, which is probably related to the surrounding topography. The values of $\sigma_{u^{*}}$ average 3-4 times larger for the Utah network compared to those for the Iowa and CASES99 networks. The greater kinetic energy of submeso motions in complex terrain is documented in [10].

\section{Daytime case}

The use of the longer time step of $10 \mathrm{~min}$ for the unstable cases leads to greater particle loss through the boundaries of the domain. Therefore, in order to maintain an adequate particle sample size, 500 new particles are randomly released after each 10-min time step throughout the simulation period from 1100 to 1500 local time.

Since the turbulence is larger scale in the daytime convective boundary layer, the range of submeso scales between the largest turbulence scales and the network width is smaller compared to the nocturnal boundary layer. However, the larger central scale of the submeso motions in the daytime boundary layer leads to larger horizontal particle displacements and therefore larger perturbations of $q$. The larger $q$ fluctuations together with stronger velocity fluctuations correspond to significantly larger horizontal diffusivities for the daytime case. For the Iowa network, the average daytime horizontal diffusivity is an order of magnitude larger than that for the nocturnal boundary layer, but the relative sampling error, $S E$, is almost order of unity. For the Utah network, the daytime horizontal diffusivity is about 2.5 times larger than the nocturnal value, on average and the relative sampling error is approximately 0.3 . Since the sampling is inadequate for the Iowa network and the small CASES99 network could not be used for daytime conditions, we are unable to form definite conclusions for the daytime case.

\section{Conclusions}

Four networks of wind observations ranging from $500 \mathrm{~m}$ width to approximately $40 \mathrm{~km}$ width were used to estimate the horizontal diffusivity due to nocturnal submeso motions. Estimation 
of horizontal gradients of scalars from nocturnal observations is problematic for much of the data due to complex spatial variation of scalars and inadequate spatial resolution. We have instead released simulated particles into the observed wind field. The particles are assigned values of a conservative variable based on the particle origin and a specified horizontal gradient. While both the coarse station spacing and particle technique limit the accuracy of the estimates of the horizontal flux (Sect. 4), the estimates do provide the first systematic evaluation of the horizontal diffusivity from observations.

The resulting horizontal diffusivities increase modestly with the range of scales of motions resolved by the data networks, but are much larger for the networks embedded in complex terrain. The averaged horizontal diffusivity increases from $<5 \mathrm{~m}^{2} \mathrm{~s}^{-1}$ for a network of $500 \mathrm{~m}$ width with relatively flat terrain to $500 \mathrm{~m}^{2} \mathrm{~s}^{-1}$ for a network a little more than $10 \mathrm{~km}$ across surrounded by modest mountains extending $200-500 \mathrm{~m}$ above the basin. The horizontal diffusivities sometime exhibit a tendency to increase with wind speed. We have avoided constructing an actual formulation for use in numerical models; however, Table 1 includes numerical information for initial guidance.

None of the networks used in this study were designed for estimation of the horizontal diffusivity. Finer station spacing is required to more completely resolve the submeso flow and detect horizontal decoupling due to cold pools. Longer operation of the network is required, particularly to obtain an adequate sample for examination of the dependence of the horizontal diffusivity on other parameters.

Acknowledgements The insightful comments of the reviewers and the discussions with Danijel Belušic are greatly appreciated. The data from Dugway Proving Ground were provided by Al Astling. Data from the Iowa network were provided by John Prueger. The CASES99 data were collected by the Integrated Surface Flux Facility of the National Center for Atmospheric Research. This material is based upon work supported by ARO Contract W911NF-08-C-0108 and NSF Grant ATM-0607842.

Open Access This article is distributed under the terms of the Creative Commons Attribution Noncommercial License which permits any noncommercial use, distribution, and reproduction in any medium, provided the original author(s) and source are credited.

\section{References}

1. Anfossi D, Oettl D, Degrazia G, Boulart A (2005) An analysis of sonic anemometer observations in low wind speed conditions. Boundary-Layer Meteorol 114:179-203

2. Anquetin A, Guilbaud C, Chollet J-P (1998) The formation and destruction of inversion layers within a deep valley. J Appl Meteorol 37:1547-1560

3. Belušić D, Mahrt L (2008) Estimation of length scales from mesoscale networks. Tellus 60:706-715

4. Etling D, Brown RA (1993) Roll vortices in the planetary boundary layer: a review. Boundary-Layer Meteorol 65: 215-248

5. Hatlee S, Wyngaard JC (2007) Improved subfilter-scale models from the HATS field data. J Atmos Sci 64:1694-1705

6. Kuettner J, Hildebrand P, Clark T (1987) Convection waves: observations of gravity wave systems over convectively active boundary layers. Quart J R Meteorol Soc 113:445-467

7. Kustas W, Li F, Jackson J, Preuger J, MacPherson J, Wolde M (2004) Effects of remote sensing pixel resolution on modeled energy flux variability of croplands in Iowa. Remote Sens Environ 92:535-547

8. LeMone MA (1973) The structure and dynamics of horizontal roll vortices in the planetary boundary layer. J Atmos Sci 30:1077-1091

9. Mahrt L (2007) Weak-wind mesoscale meandering in the nocturnal boundary layer. Environ Fluid Mech 7:331-347

10. Mahrt L, Thomas CK, Prueger JH (2008) Space-time structure of mesoscale modes in the stable boundary layer. Quart J R Meteorol Soc 135:67-75 
11. Mestayer PG, Anquetin S (1995) Climatology of cities. In: Gyr A, Rys F-S (eds) Diffusion and transport of pollutants in atmospheric mesoscale flow fields. Kluwer, The Netherlands, pp 165-189

12. Nakamura R, Mahrt L (2006) Vertically-integrated sensible heat budgets for stable nocturnal boundary layers. Quart J R Meteorol Soc 132:383-403

13. Nappo CJ (2002) An introduction to atmospheric gravity waves. Academic Press, San Diego, 297 pp

14. Nappo CJ, Miller D, Hiscox A (2008) Wave-modified flux and plume dispersion in the stable boundary layer. Boundary-Layer Meteorol 129:211-223

15. Poulos G, Blumen W, Fritts D, Lundquist J, Sun J, Burns S, Nappo C, Banta R, Newsom R, Cuxart J, Terradellas E, Balsley B, Jensen M (2001) CASES-99: a comprehensive investigation of the stable nocturnal boundary layer. Bull Am Meteorol Soc 83:555-581

16. Riley JJ, Lelong M-P (2000) Fluid motions in the presence of strong stable stratification. Annu Rev Fluid Mech 32:613-657

17. Shutts G (1998) Stationary gravity-wave structure in flows with directional wind shear. Quart J R Meteorol Soc 124:1421-1442

18. Staniforth A, Côté J (1991) Semi-Lagrangian integration schemes for atmospheric models—a review. Mon Weather Rev 119:2206-2223

19. Steeneveld G, Holtslag AAM, Nappo CJ, van de Wiel BJH, Mahrt L (2008) Exploring the possible role of small scale terrain drag on stable boundary layers over land. J Clim Appl Meteorol 47:2518-2530

20. Sun J, Lenschow DH, Burns SP, Banta RM, Newsom RK, Coulter R, Frasier S, Ince T, Nappo C, Balsley B, Jensen M, Mahrt L, Miller D, Skelly B (2004) Atmospheric disturbances that generate intermittent turbulence in nocturnal boundary layers. Boundary-Layer Meteorol 110:255-279

21. Vickers D, Mahrt L (2007) Observations of the cross-wind velocity variance in the stable boundary layer. Environ Fluid Mech 7:55-71

22. Vickers D, Mahrt L, Belušić D (2008) Particle simulations of dispersion using observed meandering and turbulence. Acta Geophys 56:234-256

23. Wilson JD, Sawford BL (1996) Reveiw of Lagrangian stochastic models for trajectories in the turbulent atmosphere. Boundary-Layer Meteorol 78:191-210

24. Wyngaard JC (2004) Toward numerical modeling in the "Terra Incognita". J Atmos Sci 61:1816-1826

25. Yagüe C, Viana S, Maqueda F, Redondo J (2006) Influence of stability on the flux-profile relationships for wind speed, $\phi_{m}$, and temperature, $\phi_{h}$, for the stable atmospheric boundary layer. Nonlinear Proc Geophys $13: 185-203$ 